\title{
Guía práctica para el cuidado y la optimización de la voz del docente
}

\author{
Olatz LARREA ESTEFANÍA \\ Universidad Pompeu Fabra \\ olatz.larrea@upf.edu
}

Recibido: $26 / 11 / 2012$

Aceptado: 23/01/2013

\begin{abstract}
Resumen
La docencia puede llegar a ser una actividad de alto riesgo para la voz, así lo demuestran múltiples estudios médicos en nuestro país. Sin embargo la consciencia de los profesionales del sector sobre la importancia de preservar su principal herramienta de trabajo aun es muy escasa. Este estudio pretende detectar los principales riesgos que afligen la salud vocal de los docentes, dentro y fuera del aula, y aportar una serie de consejos para su cuidado y optimización. Para ello, el presente estudio realiza una revisión teórica del estado de la cuestión y aporta una guía práctica en técnica vocal desde el prisma de la fisiología de la voz.
\end{abstract}

Palabras clave: voz, docencia, higiene vocal

\section{Practical Guide for the Care and Optimization of Teacher' Voice}

\begin{abstract}
Multiple medical studies demonstrate that teaching can be a high risk activity for the voice. Nevertheless the conscience of the professionals of the sector on the importance of preserving his principal tool of work is even very scanty. This study tries to detect the principal risks that afflict teacher's vocal health, inside and outside the classroom, and tries to contribute with advices to improve its care and optimization. Therefore, the present study realizes a theoretical compilation of the condition of the question and contributes with a practical guide in vocal techniques from the point of view of the physiology of the voice.
\end{abstract}

Keywords: voice, teaching, vocal hygiene

\section{Referencia normalizada}

LARREA ESTEFANÍA, Olatz (2013): "Guía práctica para el cuidado y la optimización de la voz del docente”. Estudios sobre el Mensaje Periodístico. Vol. 19, Núm. especial marzo, págs.: 271-279. Madrid, Servicio de Publicaciones de la Universidad Complutense.

Sumario: 1. Introducción. 2. Aproximación metodológica: fisiología de la voz. 3. Desarrollo. Los enemigos de la voz: principales factores de riesgo dentro del aula. 4. Conclusiones. Medidas generales de higiene vocal, postural y respiratoria. 5 . Referencias bibliográficas

\section{Introducción}

Para la mayoría de los humanos la voz es simplemente una herramienta más de expresión. Aquella sustancia sonora que nos permite comunicar y hacernos entender. No pensamos en ella de forma específica, ni tan siquiera la valoramos más allá de su función como vehículo de las palabras. Se trata de un acto inconsciente que solo se hace visible cuando falla, se altera o causa molestias. Sin embargo la voz es mucho más que eso.

"La voz es la escayola que dibuja la forma final de las palabras, la decoración que embellece o afea la superficie externa de cada idea [...] pero la voz es, ante todo, un material delicado de uso difícil que exige conocimientos técnicos profundos, entrenamiento y pasión” (Rodríguez Bravo, 2003:25). 
El manejo de la voz es en definitiva el que proporciona el marco referencial a todo lo que nos cuentan. De alguna manera, juzgamos a los individuos no sólo por su imagen sino también por su voz. Las cualidades vocales acústicas resultan tan decisivas en las relaciones sociales y públicas que condicionan en gran medida lo que los demás piensan de nosotros. (Rodero 2007: 523-542). Por tanto si la voz es decisiva en nuestra vida cotidiana, más aun lo será en todas aquellas profesiones que como señala Le Huche (1994) tienen la "obligación socio-profesional de hablar". Resulta lógico pensar que cualquier profesional que ocupe la mayor parte de su actividad hablando debe cuidar su principal herramienta de trabajo. Sin embargo, la consciencia sobre la necesidad de preservar adecuadamente nuestro aparato vocal no está tan extendida como quisiéramos entre docentes, abogados, radiofonistas, presentadores y oradores. El mal uso de la voz y el desconocimiento de la técnica vocal es la culpable de gran parte de las patologías que afligen a estos profesionales, que la mayoría de las veces no admiten que la voz sea una herramienta fundamental en su día a día y por tanto no consideran que deban pedir ayuda profesional o ponerse en tratamiento cuando ésta presenta alteraciones. (Russell et al., 1998; Smith, Kirchner, et al., 1998).

"A pesar de las cifras que indican que un gran número de profesores padecen patologías vocales, en ocasiones, los docentes, se muestran poco dispuestos a dedicar tiempo (en horario laboral) a citas médicas o temen que el médico pueda recomendarles reducir el empleo de su voz en el trabajo, dejar de dar clases totalmente e incluso cambiar de profesión" (Nelson et al. 2004: 281-93).

Numerosos estudios llevados a cabo en nuestro país (Herrero, 1986; Esteve et al, 1991; García Calleja, 1991) han demostrado que entre las principales causas de absentismo laboral de los docentes, se encuentran las patologías de la voz. El informe del Consejo Escolar del Estado del año 2000 recoge el "aumento del absentismo laboral, especialmente en el segundo trimestre del curso, con graves repercusiones en el rendimiento de los alumnos". No en vano las enfermedades otorrinolaringológicas suponen la tercera causa de las bajas laborales entre los docentes.

La Organización Internacional del Trabajo (OIT) sitúa al profesorado como la primera categoría profesional bajo riesgo de contraer enfermedades profesionales de la voz. Sin embargo hasta el año 2006 no se reconoció esta patología como una enfermedad profesional, cuando finalmente se registró como enfermedad profesional, con la entrada en vigor del Real Decreto 1299/2006, la única patología descrita fueron "los nódulos de las cuerdas vocales a causa de los esfuerzos sostenidos de la voz por motivos profesionales". Previamente, multitud de estudios llevados a cabo en Estados Unidos (Lejska, 1967; Marks, 1985; Pekkarinen, Himberg, \& Pentti, 1992; Russell, Oates, \& Greenwood, 1998; Smith, Gray, Dove, Kirchner, \& Heras, 1997; Titze, Lemke, \& Montequin, 1997; Smith, Kirchner, Taylor, Hoffman, \& Lemke, 1998; Smith, Lemke, Taylor, Kirchner, \& Hoffman, 1998), habían demostrado que la docencia es una actividad de alto riesgo para la salud vocal con una prevalencia en continuo crecimiento tanto en la población profesional como en la general, aunque en esta última en menor medida. Junto a estos resultados, las investigaciones de Nelson et al. (2004: 281-93) advirtieron del riesgo específico al que se enfrenta el colectivo femenino ante las patologías derivadas de su profesión, estableciendo que "ser mujer, 
entre 40 y 59 años, con una experiencia docente de más de 16 años, y con un historial familiar con antecedentes patológicos está directamente relacionado con haber padecido alteraciones vocales en el pasado o padecerlas en el presente".

Sin embargo, a pesar de las múltiples voces lanzadas desde el campo médico para advertir a los docentes sobre la importancia del cuidado de la voz, la producción de literatura científica sobre el tema aun es escasa.

Para remediar esta carencia el presente artículo se propone como único objetivo, detectar los principales factores de riesgo para la salud vocal del docente dentro y fuera del aula y aportar una serie de consejos para su cuidado y optimización. Para ello se realizara una aproximación teórica a la técnica vocal desde el prisma de la fisiología de la voz. Comenzaremos por entender el funcionamiento del aparato fonador, principal instrumento creador de la voz.

\section{Aproximación metodológica: fisiología de la voz}

Existen varias aproximaciones teóricas que estudian las patologías de la voz (médica, psicológica, quiropráctica...), este estudio parte del prisma de la de la fisiología de la voz para tratar de explicar de forma práctica las bases de la técnica vocal. Para ello se ha realizado una revisión de artículos, material docente y literatura producida desde los departamentos de prevención de riesgos laborales de las diferentes comunidades autónomas y se han sintetizado en una guía práctica de consejos sobre la higiene vocal la respiración y la postura. Este artículo parte del estudio de la técnica vocal como principal estrategia de prevención de los trastornos de la voz y también como posible alternativa para recuperar la voz enferma a través de la reeducación de su uso y producción.

Partimos de la premisa fundamental de que el uso adecuado de la voz implica un aprendizaje específico. "Es necesario conocer el mecanismo de la fonación y los recursos de la voz, para tomar conciencia de los propios hábitos, para corregirlos mediante el ejercicio y la automatización de los mecanismos más adecuado."(Calatrava González, Junta de Andalucía). Entre la población docente existe un alto porcentaje de profesorado que utilizan mal la voz y realizan esfuerzos poco eficaces para ser escuchados. Para empezar a enmendar estas conductas involuntarias debemos comprender que la voz es el sonido resultante de la puesta en marcha de casi nuestro cuerpo al completo.

Aunque pudiéramos pensar que en la emisión de la voz únicamente entra en juego el aparato fonador nos equivocamos, ya que en este ejercicio pueden llegar a intervenir un elevado número de músculos y cavidades corporales. Nuestro cuerpo tiene incorporado un instrumento de viento bien afinado que es capaz de producir sonido pero que no funcionaría sin la colaboración del resto del cuerpo. Para emitir sonidos, ese instrumento se nutre de tres elementos básicos: el aire, la vibración y la resonancia. De esta manera resulta lógico comprender que el proceso de emisión de sonidos empieza con el aire inspirado desde los pulmones y comprende tres fases en las que intervienen diferentes músculos, según el médico foníatra Roques (2007):

- El sistema respiratorio (musculatura respiratoria abdominal y torácica, pulmones, bronquios, tráquea) que se encargará de producir un soplo de aire controlado para la correcta producción de sonido. Actúa como fuelle o bomba de aire. 
- La laringe como estructura compleja formada de cartílagos, articulaciones y músculos, será la encargada de la vibración que se produce al recibir aire a presión procedente del soplo pulmonar generando así ruido. Sus principales activos son las cuerdas vocales y la membrana mucosa que se abren y cierran durante la espiración produciendo el sonido natural de la voz.

- Las cavidades de resonancia (zona faríngeo-bucal, cavidad nasal y esqueleto óseo de la cara). Zonas en las que el sonido laríngeo resultante de la vibración de las cuerdas vocales se amplifica, se modela y se mejora antes de salir al exterior del organismo. Todo ello controlado por el sistema auditivo y el sistema nervioso central. Por todo este proceso las logopedas Albar y Ruiz del Árbol definen la voz como "el aire que adquiere sonido al pasar entre las cuerdas vocales y se amplifica en la caja de resonancia" (Eduvoz-formación). Sin embargo, cuando todo este circuito finaliza, la voz resultante de nuestro cuerpo no es siempre la misma, varía según la situación comunicacional en la que se encuentre el hablante y los hábitos adquiridos que emplee en la producción de su voz. Por ello, los foniatras distinguen entre tres usos diferentes de la voz (Roques, 2007):

- La voz conversacional: se trata de una voz no proyectada, de uso diario. Es la voz que el docente utiliza en casa, con sus familiares, en conversaciones habituales, en las que no habla para una audiencia sino para un grupo íntimo o reducido. Se trata de situaciones relajadas en las que el objetivo final es comunicar, por lo que no es necesario impostar la voz ni aplicar una técnica vocal depurada. Basta con hacer un uso razonable de la voz para no padecer molestias.

- La voz proyectada: "en este caso se pretende, con nuestra acción vocal, actuar o influir sobre otro u otros" (Roques, 2007). También llamada voz directiva es, por lo general, la voz que usan los docentes en el aula. Habitualmente se produce en un ambiente de ruido o alboroto, en el que el hablante necesita hacerse oír, por lo tanto se genera una situación de estrés o de tensión. En estos contextos el docente necesita imprimir a su voz más intensidad y el cuerpo entero se vuelca en esa tarea. Esta tensión física y psicológica provoca que la voz proyectada no fluya de manera sana. Por esta razón, para conseguir una voz proyectada sin sobreesfuerzos es preciso un entrenamiento en técnica vocal, además de la adopción de medidas de higiene vocal.

- La voz de apremio: Se trata de la voz resultante del continuo mal uso y sobreesfuerzo de la misma. En ocasiones, la voz conversacional puede convertirse en voz de apremio tras un uso viciado prolongado en el tiempo. Es el resultado de una tensión muscular continuada, una incorrecta respiración y vicios posturales. Normalmente se manifiesta con dolores en la faringe y sensaciones de cansancio vocal y fatiga. El resultado es una voz estridente e incontrolada.

De esta manera, la proyectada será la voz de trabajo del docente; aquella que le permita llegar a todo al auditorio. Sin embargo no se debe confundir la voz proyecta con hablar a gritos, sino que se debe intentar llegar a la audiencia sin forzar la voz. Los docentes exponen sus voces a largas sesiones docentes, en ambientes no siempre propicios (sequedad, polvo, ruido) y en ocasiones cuando la voz ya está afectada por catarros o ronqueras; en definitiva indicios de una voz enferma. 
Por estas razones, conseguir una voz proyectada sana comienza por entrenar la voz en la técnica vocal para evitar las patologías derivadas de un mal uso, seguir las medidas de higiene vocal necesarias, minimizar los factores ambientales que la afligen e iniciarse en la reeducación postural y respiratoria que permitan al hablante conservar la voz sana, o en el peor de los casos, saber corregir su estado cuando comienza a dar síntomas de estar enferma. Por todo ello,

"La voz profesional es el resultado del esfuerzo físico de unos órganos, pero regulados por un tiempo, un ritmo y las características impuestas por la situación emotiva y mental que vive la persona, utilizando las técnicas respiratorias y vocales adecuadas, de manera que responda satisfactoriamente y resuelva de modo correcto la situación vocal que presente el auditorio en sus diferentes modalidades" (Bustos, 2003)

\section{Desarrollo. Los enemigos de la voz: principales factores de riesgo dentro del aula}

Existen varios factores que han sido descritos como potenciales enemigos de la voz del docente, a continuación describimos los más habituales dentro del aula, y proponemos algunas medidas desde el campo de la higiene vocal, para prevenirlos.

Las condiciones de ventilación, humedad y temperatura del aula serán factores a tener muy en cuenta ya que el lugar de trabajo es un posible enemigo de la voz si no está bien acondicionado. Las corrientes de aire pueden afectar al sistema respiratorio y por tanto a la fonación. La humedad es un problema en las aulas tanto por exceso como por defecto. Esta última situación suele ocasionarse en invierno cuando la calefacción está encendida. La principal consecuencia es que el ambiente seco dificulta el mantenimiento de los niveles de lubricación de las cuerdas vocales necesarias para la fonación. En estos casos es fundamental beber agua continuadamente. El exceso de humedad también es un problema que aparece normalmente en las clases que se realizan en el exterior. Por otro lado, una correcta ventilación del aula es fundamental para eliminar cualquier tipo de posible elemento patógeno que pueda afectar a la voz.

El ruido es otro de los factores que agreden la voz del docente. En ocasiones en el aula se cuelan ruidos procedentes de la calle (coches, obras, alarmas, ambulancias...) o que proceden del propio edificio (mala distribución de espacios para clases, insonorización insuficiente, aulas excesivamente grandes con reverberación...). Además de los ruidos más comunes que son los que generan los alumnos. Este alboroto obliga al profesor a subir el volumen de su voz y por tanto a forzarla para imponerse, hacerse oír o simplemente llamar la atención. Son reacciones inconscientes de los docentes ante situaciones de descontrol. Desde la junta de Extremadura (Dirección General de Personal docente) advierten de que el ruido ambiental normal en las aulas está registrado generalmente en torno a los $55 \mathrm{~dB}$. El docente intensifica espontáneamente su voz $15 \mathrm{~dB}$ por encima del ruido, lo que supone que su voz se sitúa a unos $70 \mathrm{~dB}$ mientras que la intensidad normal establecida para la voz conversacional es de $65 \mathrm{~dB}$. De modo que se produce un riesgo potencial para las cuerdas vocales. La solución ante estos casos es utilizar siempre algún sistema de amplificación de audio; el micrófono es el más práctico pero también sirve cualquier otro material auxiliar que permita al docente descansar la voz (power point, altavoces, vídeos...) 
La propia tarea del docente puede ser en esencia la base de los problemas de fonación. La exposición a largas jornadas de uso de voz sin un correcto calentamiento es un riesgo. Los horarios de clase también pueden ser desfavorables incrementándose el riesgo a primera hora de la mañana y después de comer.

"Tras despertarnos, la actividad orgánica está regida por el sistema simpático (la parte del sistema nervioso que gobierna la vida vegetativa, independiente de la voluntad) lo que dificulta la actividad de las cuerdas vocales. Después de comer se activa la digestión (función regulada, al igual que la fonación, por el nervio vago) apareciendo sopor, que induce a una fonación más costosa, que se agrava ante la dificultad del descenso del diafragma, debido a un aumento del volumen del estómago" (Dirección General de Personal Docente, Junta de Extremadura: 4)

Por todo ello mantener unos hábitos saludables en el estilo de vida que contemplen una alimentación sana y ejercicio físico frecuente ayudarán a corregir tensiones y a ser menos vulnerables ante las patologías de la voz. Además mitigarán las consecuencias físicas del sedentarismo y nos dotarán de un tono muscular adecuado para afrontar el desgaste energético del aula.

La tensión presente en el desarrollo de la tarea docente es un riesgo fundamental. Existen varios estudios (Federación de Sindicatos Independientes de la enseñanza) que apuntan a los primeros años de ejercicio de la profesión como los de mayor riesgo vocal (estrés del principiante). El joven docente aun no ha aprendido a dosificar sus esfuerzos y tiende a sobrecargar la voz sin planificar su energía. Sin embargo, también los docentes experimentados sufren las consecuencias del estrés al enfrentarse a una nueva asignatura sin ser conscientes del esfuerzo físico y psicológico al que están sometidos. Para mejorar estas situaciones es recomendable descansar adecuadamente (aproximadamente 8 horas de sueño), vigilando las interrupciones del sueño por desvelo o malas posturas. Los estiramientos pueden ayudar a corregir las consecuencias de un mal descanso, pero en caso de persistencia se debe consultar con los especialistas.

\section{Conclusiones. Medidas generales de higiene vocal, postural y respiratoria}

Además de las recomendaciones para el cuidado de la voz en el aula existen otras medidas de higiene vocal que los docentes pueden aplicar en su vida fuera del centro. A continuación citamos las más relevantes:

- Aprender a detectar los primeros síntomas de fatiga en la voz, así como no pasar por alto signos prolongados de ronqueras, catarros o procesos gripales leves es fundamental. Consultar con el especialista en caso de que persistan entre 10 y 15 días.

- Vigilar nuestro uso vocal en ambientes ruidosos (bares, discotecas, hablando por teléfono...) así como en medios de transporte (coche o moto) que tienden a hacernos forzar la intensidad de la voz.

- De la misma manera que las recomendaciones de salud general sirven para toda la población, también se deben aplicar a los docentes: el tabaco, el alcohol, las bebidas frías y el exceso de cafeína ponen en riesgo nuestro aparato vocal. Debemos evitar exponernos al humo, al polvo o al aire acondicionado, así como a cambios bruscos de temperatura. 
- Es muy importante mantener hidratada la mucosa de la laringe, por lo que se recomienda ingerir preferiblemente agua sin gas (una media de 2 litros diarios). La postura de nuestro cuerpo influye en la calidad vocal. Por ello, de la misma manera que la acción de andar, la de hablar es una función motora que debe ser realizada de forma responsable y sin sobrecargas. De este modo:

- Debemos prestar atención a la postura que adoptamos durante el ejercicio de la fonación y mantener un buen equilibrio postural durante el trabajo. Por ello se deben evitar las posturas poco naturales (en cuclillas o agachados). Es recomendable impartir las clases con la columna recta y el cuerpo relajado (ya sea en pie o sentado)

- Debemos evitar hablar con la boca entrecerrada y poco articulada si lo que se pretende es proyectar la voz. Además, el esfuerzo vocal debe impulsarse y controlarse desde la zona abdominal, evitando empujar desde el cuello, pues tensamos así las cervicales y los hombros

- Durante la práctica de ejercicios específicos de técnica vocal se recomienda utilizar ropa cómoda y poco ajustada.

- Una vez que finalice la jornada laboral, de la misma manera que se aplica tras el ejercicio físico, debemos hacer un periodo de descanso, tanto vocal como psicofísico, antes de reiniciar cualquier otra actividad.

- Las situaciones de gran tensión muscular, de estrés, de tensión emocional, de enfado, etc., no favorecen al sistema fonador que se resiente de la tensión y rigidez generalizada. La técnica respiratoria, sumada a la higiene vocal y a la educación postural, es fundamental para la una correcta fonación. Por esta razón se recomienda:

- Practicar una respiración costo-diafragmática, aquella que al inspirar dirige el chorro de aire al fondo de los pulmones. El diafragma desciende por la presión del aire y el abdomen asciende. Se reconoce por la expansión de las costillas inferiores hacia los laterales.

- En cualquier caso se debe evitar contener la respiración durante breves periodos (mientras se piensa...) O realizar una mala gestión del aire durante la frase, agotando su reserva a mitad de una explicación. Para ello se recomienda hablar durante la aspiración del aire y hacer pausas para la inspiración.

La falta de formación es la principal causa de problemas vocales. Si se tiene en cuenta el número de horas que trabajan los docentes con su voz y la importancia para el desarrollo profesional de poder usarla sin descanso, resulta evidente que la docencia es una profesión con un alto índice de riesgo vocal. Por esta razón, la formación específica en este campo es básica para un ejercicio saludable de la práctica docente.

\section{Referencias bibliográficas}

BUSTOS, Inés (2003): La voz. La técnica y la expresión. Barcelona, Paidós.

CALATRAVA GONZALEZ, Ma Ángeles: Educación de la voz y prevención de sus patologías. Seguridad y salud laboral docentes. Junta de Andalucía. Disponible en http://www.juntadeandalucia.es/educacion/portal/com/bin/salud/contenidos/Bi- 
bliotecaVirtual/Documentos/CursoEducacionVoz/1158924432547_curso_de_educacin_de_la_voz.pdf. Consultado el 6 de Noviembre de 2012

CONSEJO ESCOLAR DEL ESTADO (2000): "Las enfermedades profesionales". Prevención de Riesgos Laborales. Madrid, Ministerio de Educación Cultura y Deporte. Consultado el: 30 de octubre de 2012

DIRECCIÓN GENERAL DE PERSONAL DOCENTE. Servicio de Salud y Riesgos Laborales de Centros Educativos. Consejería de Educación. Junta de Extremadura. Disponible en http://profex.educarex.es/profex/Ficheros/RiesgosLaborales/05_Problemas_Voz.pdf. Consultado el: 27 de septiembre de 2012.

EDUVOZ: Materiales docentes. Disponible en http://www.eduvozformacion.com/. Consultado el 27 de septiembre de 2012.

ESTEVE, José Manuel et al (1991): "La salud de los profesores. Evolución del 1982 a 1989". Revista Cuadernos de Pedagogía, 192, 61-67.

FEDERACIÓN DE SINDICATOS INDEPENDIENTES DE LA ENSEÑANZA: Fundación para la prevención de Riesgos Laborales. Disponible en http://fsieprlinfantil.es/?page id=19

GARCÍA CALLEJA, Manuel (1991): "Enfermedades del profesorado. Análisis y prevención". Revista Cuadernos de Pedagogía, 192, 67-72.

HERRERO, Ma Teresa (1986): “Aspectos sanitarios públicos en los problemas de voz en docentes". Actas del XIV Congreso Nacional de A.E.L.FA., Burgos, 105-110 en NUÑO PEREZ, J. et al. (1996): "Problemas de la voz en el profesorado". Revista interuniversitaria de formación del profesorado, 26, 33-42.

LE HUCHE, François, ALLALI, André (2004): La voz. Barcelona, Masson.

LEJSKA, V. (1967): Occupational voice disorders in teachers. Pracovini Lekarstvi, 19, 119-121.

MARKS, J. B. (1985): "A comparative study of voice problems among teachers and civil service workers". Unpublished master's thesis. Minneapolis, University of Minnesota.

NELSON, Roy; MERRILL, Ray M; THIBEAULT, Susan; PARSA, RAHUL, A; et al (2004): "Prevalence of Voice Disorders in Teachers and the General Population". Journal of Speech, Language, and Hearing Research, 47, 281-93

PEKKARINEN, E., HIMBERG, L., \& PENTTI, J. (1992): "Prevalence of vocal symptoms among teachers compared with nurses: A questionnaire study". Scandinavian Journal of Logopedics and Phoniatrics, 17, 112-117.

PRECIADO, Julián el al. (2005): "Incidencia y prevalencia de los trastornos de la voz en el personal docente de La Rioja". Acta Otorrinolaringológica, 56, 202-210

RODERO, Emma (2007): "Caracterización de una correcta locución”. Estudios sobre el mensaje periodístico, 13, 523- 542. Madrid, Servicio de Publicaciones de la Universidad Complutense. 
RODRÍGUEZ BRAVO, Ángel (2003): La voz, en RODERO, Emma: Locución Radiofónica. Madrid, IORTV, pág. 25.

ROQUE CALAMITA, Manuel (2007): "La voz del docente: voz proyectada", en I Plan andaluz de salud laboral y prevención de riesgos laborales del personal docente de los centros públicos dependientes de la Consejería de Educación. Acciones 15 y 18. Disponible en http://www.omerique.net/twiki/pub/CEPCA3 /ActividadFormacion081106CU043/Lavozdeldocente_VozProyectada.pdf Consultado el: 27 de Septiembre de 2012.

RUSSELL, A., OATES, J., \& GREENWOOD, K. M. (1998): "Prevalence of voice problems in teachers". Journal of Voice, 12, 467-479.

SANCHEZ RANCHAL, Antonio y VAQUERO ABELLAN, Manuel (2008): "Protocolo para la vigilancia de la salud del profesorado con atención a la enfermedad profesional". Scielo, vol. 54 no. 211. Disponible en:http://dx.doi.org/10.4321 /S0465546X2008000200007 Consultado el 27 de septiembre de 2012.

SMITH, E., GRAY, S. D., DOVE, H., KIRCHNER, H. L., \& HERAS, H. (1997): "Frequency and effects of teachers' voice problems". Journal of Voice, 11, 81-87.

SMITH, E., KIRCHNER, H. L., TAYLOR, M., HOFFMAN, H., \& LEMKE, J. H. (1998): "Voice problems among teachers: Differences by gender and teaching characteristics". Journal of Voice, 12, 328-334.

SMITH, E., LEMKE, J., TAYLOR, M., KIRCHNER, H. L., \& HOFFMAN, H. (1998): "Frequency of voice problems among teachers and other occupations". Journal of Voice, 12, 480-488.

TITZE, I. R., LEMKE, J., \& MONTEQUIN, D. (1997): "Populations in the U.S. workforce who rely on voice as a primary tool of trade: A preliminary report". Journal of Voice, 11, 254-259.

\section{Olatz LARREA ESTEFANÍA}

Universidad Pompeu Fabra

Personal Investigador en Formación

olatz.larrea@upf.edu 